\title{
Narrativas de Profesionales Chilenos Sobre sus Trayectorias Laborales: La Construcción de Identidades en el Trabajo
}

\section{Narratives of Chilean Professionals About Their Career Paths: The Construction of Identities at Work}

\author{
Álvaro Soto \\ Universidad Alberto Hurtado
}

\begin{abstract}
Se reporta un estudio cualitativo sobre las trayectorias laborales móviles y su relación con las formas de construcción identitaria características del trabajo contemporáneo en Chile. Como una forma de acercamiento heurístico a las ofertas de sentido y a las lógicas de significación dominantes en las empresas actuales, se analizaron las narrativas de nueve profesionales "de elite" de organizaciones inmersas en el mercado de trabajo flexible acerca de sus propias trayectorias laborales. El diseño metodológico cualitativo se basó en el modelo de la grounded theory. La información generada a partir de entrevistas individuales en profundidad se analizó siguiendo el método de la comparación continua, logrando identificar sentidos compartidos en torno a la actividad del trabajo, la orientación de las trayectorias y los vínculos generados en el trabajo, que marcan continuidades a nivel subjetivo y constituyen pilares de construcción de identidades laborales. Estas se estructuran en torno a experiencias de trabajo centradas casi exclusivamente en el corto plazo y representaciones de las trayectorias basadas en la movilidad dentro de un mercado de trabajo abierto.
\end{abstract}

Palabras clave: trayectorias laborales, identidad, trabajo, profesionales, flexibilidad

\begin{abstract}
This article examines the phenomenon of mobile labor careers and analyzes its relationship with characteristic forms of identity construction in the contemporary workplace in Chile. In order to approach the meanings and dominant logics in current enterprises from an heuristic perspective, an analysis was carried out of the narratives regarding the careers of nine "high-level" professionals within organizations embedded in the flexible labor market. The methodological design was based on the grounded theory model. The information generated from individual in-depth interviews was analyzed following the method of continuous comparison. Using this method, the authors identified shared meanings about labor activity, the orientation of the career paths of the interviewees, and the social bonds generated at work, which mark subjective continuities and constitute foundations for the construction of labor identities. They are structured almost exclusively on short-term work experiences and representations of career paths based on mobility within an open labor market.
\end{abstract}

Keywords: labor careers, identity, work, professionals, flexibility

\section{Antecedentes}

\section{La Permanente Pregunta en Torno a las Identidades en el Trabajo}

En torno al concepto de identidad confluyen tanto procesos constitutivos de subjetividad -el mundo interno del sujeto- como dinámicas de relación social desarrolladas en determinadas situaciones y espacios de poder. Esta doble dimensión del constructo ha sido descrita, por ejemplo, en los estudios culturales, en los que la identidad se ha entendido como el proceso de sujeción a prácticas discursivas —y a sus disposiciones-en espacios sociales de interacción, es

Álvaro Soto Roy, Facultad de Psicología, Universidad Alberto Hurtado, Santiago, Chile.

Este estudio fue posible gracias al financiamiento del Fondo Nacional de Ciencia y Tecnología (FONDECYT), Proyecto $N^{\circ} 11090154$.

La correspondencia relativa a este artículo debe ser dirigida a Álvaro Soto Roy, Facultad de Psicología, Universidad Alberto Hurtado, Almirante Barroso 26, Santiago, Chile. E-mail: asoto@uahurtado.cl 
decir, al espacio de encuentro y confrontación entre prácticas sociales y los procesos que permiten construirse subjetivamente a partir del "decirse" (Hall, 2003).

Respecto de los procesos identitarios desarrollados en espacios de trabajo, destacan los aportes que desde la sociología ha realizado Claude Dubar (1991, 1992, 2000), quien sitúa los procesos de construcción identitaria en la superposición de procesos de transacción objetiva y subjetiva. La identidad laboral resulta de la confrontación entre la demanda y la oferta de identidades posibles, lo que plantea los problemas de la desigualdad en los medios sociales para obtener reconocimiento y de las capacidades del sujeto para atribuir un sentido durable a su experiencia (Dubar, 1991; Sainsaulieu, 1977).

La teoría organizacional y los estudios de administración han abordado el fenómeno identitario en el trabajo desde un enfoque narrativo-discursivo, en el que la identidad da cuenta del trabajo de reparación, mantención y reforzamiento de las construcciones que dan sentido de coherencia y distinción a las personas (Alvesson \& Willmott, 2002; Sveningsson \& Alvesson, 2003). La identidad laboral daría cuenta del difícil y nunca acabado proceso de interpretación, integración y estructuración de las propias experiencias en el trabajo, representando un patchwork de autopercepciones que ofrece continuidad biográfica, consistencia entre las diferentes esferas de la vida y atribución de causalidad de los eventos personalmente relevantes (Grote \& Raeder, 2009).

Complementando el enfoque narrativo-discursivo y relevando la dimensión relacional de la identidad laboral, Down y Reveley (2009) proponen atender a las interacciones cara a cara del sujeto en el trabajo, en cuanto procesos de auto-verificación y compensación de las identidades narrativas inestables del trabajo contemporáneo. Se apela a una mirada dramatúrgica de la identidad (Goffman, 2001, 2003), centrada en la presentación y despliegue de sí en la interacción que busca causar — consciente o inconscientemente- determinadas impresiones en los demás.

El trabajo que se presenta supone que el análisis de las formas de construcción identitaria en el trabajo contemporáneo permite acceder a la comprensión de evoluciones sociales más generales de la sociedad (Alvesson, Ashcraft \& Thomas, 2008). La identidad laboral se construye en el contexto de funcionamiento del mercado del trabajo, de la organización productiva y de los sistemas de gestión. Estos escenarios del trabajo contemporáneo, caracterizados por la individualización y la ruptura permanente (Soto, 2009; Soto, Espinoza \& Gómez, 2008), definirían identidades laborales basadas en la adhesión intermitente a colectivos múltiples, en los que los sujetos gestionarían recursos de identificación de manera diversa a partir de estrategias fundadas en los principios de movilidad, opción individual y realización personal (Dubar, 2000).

\section{Construcción Identitaria y Movilización Subjetiva en la Empresa}

Los modelos de gestión de personas propios de la empresa contemporánea buscan desplazar el tradicional control externo de la conducta por formas de control subjetivo basadas en la identificación del sujeto con la empresa, sus valores y su cultura. La ideología managerial (Le Goff, 1995), propia al nuevo espíritu del capitalismo (Boltansky \& Chiapello, 1999), concibe a la empresa como fuente de valores y espacio de integración social, que ofrece posibilidades de desarrollo y realización a partir de la utilización de todas las capacidades de la persona en contextos laborales autónomos. En vez de coerciones directas, se apela al profesionalismo y a instrumentos concretos de gestión (e.g., metas y bonos) que implican la aceptación lúcida y pragmática de la exclusión y de la marginalización como sanción a la no-movilización (Courpasson, 1997, 2000).

Se desarrollarían así procesos de construcción identitaria en los que la realización personal se juega en torno al proyecto de la empresa, mezcla de cálculo estratégico y de impregnación cultural, caracterizados por la transacción subjetiva de implicación personal en el trabajo a cambio de la seguridad del empleo y de la progresión probable de la carrera en la empresa (Dubar, 2000). 
En esta búsqueda de movilización subjetiva, la empresa utiliza saberes psicológicos que garantizan comportamientos de autonomía, responsabilidad y competencia, basados en repertorios interpretativos vinculados a la autorrealización, el progreso y la flexibilidad en el trabajo (García \& Carvajal, 2007). Estos buscan suscitar el sentimiento de propiedad y autodisciplina a través de procesos de naturaleza narcisista que aseguran la canalización de la energía psíquica hacia la identificación con la empresa, el trabajo productivo y la aceptación de valores organizacionales (Aubert \& De Gaulejac, 1991; Enriquez, 1997a, 1997b), apelando a la sensación de formar parte de una elite profesional que proveería seguridad existencial en el ejercicio de roles caracterizados por la ambigüedad y el permanente cuestionamiento (Alvesson \& Robertson, 2006).

Ahora bien, tal como proponen Kosmala y Herrbach (2006), si bien los sujetos acatarían las normas y valores propios de las tecnologías del yo, al mismo tiempo desarrollarían dinámicas de distanciamiento subjetivo que les permiten jugar con ellas o privilegiar unas sobre otras, a partir de una actitud parcial de cinismo y de disposición al goce, que funciona como mecanismo protector en un ambiente postmoderno que exige el endurecimiento de las exigencias del yo.

\section{La Construcción Identitaria en el Marco de Trayectorias Móviles}

La mayor movilidad que caracterizaría a las trayectorias laborales actuales respecto de las formas estables en que antiguamente se desarrollaban las carreras laborales llevaría a la configuración de trayectorias laborales cambiantes y flexibles, también denominadas de mosaico o de Proteo (por el dios griego Proteo, asociado a la transformación permanente).

Esta movilidad puede explicarse, en un primer nivel, por el interés de las propias empresas por asegurar su flexibilidad en entornos cambiantes e impredecibles, a partir de prácticas de gestión del empleo basadas en temporalidades breves y en el cambio dotacional permanente (Martínez, De Cuyper \& De Witte, 2010). A esto se añade un discurso que promueve el rol activo de los sujetos en la configuración de sus propias trayectorias laborales, centrado en el principio de la empleabilidad, el cual enfatiza la iniciativa y la responsabilización del individuo (Gazier, 2003). Desarrollar la propia empleabilidad exige a los sujetos una amplitud de competencias de tipo relacional, estratégico y metacognitivo, transferibles a diferentes empleadores y focalizadas en la individualización, que se traducen en proactividad, claridad de objetivos y altos grados de conciencia de sí mismo (Enríquez \& Rentería, 2007; Rentería \& Malvezzi, 2008).

En un segundo nivel de explicación, las trayectorias móviles pueden ser consideradas como la expresión de nuevas representaciones de los sujetos, que les hace más disponibles a la flexibilidad. La psicología organizacional norteamericana ha tendido a proponer la tesis del cambio cultural, donde la movilidad se explicaría a partir de la búsqueda de mayor realización y satisfacción en el trabajo. Los sujetos estarían más disponibles a la flexibilidad en la medida que tienen más clara su vocación y sus objetivos, así como mayor conciencia de sus capacidades (Hall \& Chandler, 2005). Surgirían así nuevas formas de contrato psicológico en la empresa, entendido como las expectativas generales de reciprocidad que construye el propio sujeto frente a la organización (Morrison \& Robinson, 1997; Rousseau, 1995). Concretamente, existiría un desplazamiento desde las clásicas lógicas de contrato psicológico de tipo relacional — basadas en compromisos de largo plazo - hacia lógicas de tipo transaccional, focalizadas en intereses de corto plazo y permanentemente abiertos a (re)negociación, que implican obligaciones específicas y un involucramiento limitado por parte del sujeto (Alcover, 2002).

Estudios recientes con grandes muestras evidencian que las carreras flexibles tienden a acrecentarse en el mercado del trabajo y particularmente en aquellos sectores más dinámicos. Sin embargo, también constatan que la evaluación de los trabajadores acerca de la flexibilidad dista enormemente del ideal teórico de la autorrealización en el trabajo. Grote y Raeder (2009) constatan que solo una minoría de trabajadores se mostraría abiertamente disponible a la flexibilidad y en ellos se observarían vivencias complejas que mezclan la sensación de éxito de carrera y de fracaso a nivel personal. Prevalecen identidades profesionales críticas y poco 
disponibles a la flexibilidad, basadas en la insatisfacción frente a su situación laboral flexible y a la percepción de demanda excesiva de flexibilidad por parte de la empresa. Por su parte, Elchardus y Smits (2008) constatan que los modelos de carrera flexible tienden a desvanecerse rápidamente frente a la prueba de las transiciones vitales y a la relación entre el trabajo y la vida familiar. La mayor parte de los trabajadores mostraría una baja disposición a desarrollar carreras flexibles, buscando promociones en un mismo sector o mantenerse en el empleo. La disposición favorable a la flexibilidad, por el contrario, respondería a estrategias de adaptación a la precariedad y a lógicas (minoritarias) cercanas al ideal de carreras flexibles.

Los antecedentes planteados permiten proponer que los procesos contemporáneos de construcción identitaria en el trabajo se mueven entre dos polos, uno que invita a la identificación y a la implicación de los sujetos con la empresa y otro que promueve la individualización a partir de la responsabilización en torno a la propia trayectoria y la movilidad permanente. Estos polos estarían ciertamente en tensión, lo que ha llevado a Dubar (2000) a proponer la tesis de la crisis de identidades, propia al trabajo contemporáneo.

\section{Las Posibilidades de Construcción Identitaria en un Mercado de Trabajo Segmentado}

Las posibilidades de acceso a formas valorables y atractivas de identidad en el trabajo se hacen críticas en escenarios de empleo fuertemente segmentados (Everaere, 1999; Gadrey, 1996; Maruani \& Reynaud, 1993), más aún en Chile, donde se ha evidenciado una distribución particularmente desigual de los ingresos (Contreras, 1999; Infante \& Sunkel, 2009), fundada en prácticas de empleo flexible (López, 2008; Soto et al., 2008), caracterizadas por la externalización y la precarización (Abramo, Montero \& Reinecke, 1997; Echeverría, 2009; Raineri, 2001).

Los estudios disponibles acerca de las trayectorias laborales en Chile se focalizan en contextos de alta precariedad laboral, constatando desigualdades en las oportunidades que se ofrecen a hombres y mujeres en el mercado del trabajo (Mauro, 2004; Mauro \& Yáñez, 2005) y fuertes exigencias para saltar entre empleos dependientes e independientes (Acuña, 2005, 2008). Existen, sin embargo, pocas evidencias empíricas acerca de las lógicas de construcción identitaria desarrolladas por el segmento dotacional "privilegiado". Ghiardo y Dávila (2008) constatan discursos de jóvenes chilenos sobre sus trayectorias, basados en la aceptación de las reglas del juego del sistema productivo, la individualidad y la competencia. Si bien se valora la movilidad, se rechaza la desprotección y la pérdida de garantías del empleo. Por su parte, Sisto y Fardella (2009) analizan narrativas sobre trayectorias de profesionales en condiciones de empleo flexible, que coinciden en organizarse a partir de versiones de sí mismo/a en cuanto sujeto que se conduce por decisiones individuales y proyectos personales que justifican la asunción de riesgos para el éxito profesional. Los autores ponen especial atención en la existencia de fisuras en estos relatos, que tienden a contradecir estas narrativas canónicas, las cuales son rápidamente reducidas a través del trabajo de control narrativo.

Entender los pilares de sentido en las trayectorias de profesionales "exitosos" no solo permite entender las lógicas de construcción social de una elite sino, también, identificar ejes de significación que se presentan al resto de la población como deseados y/o posibles de desarrollar en el trabajo, es decir, las ofertas identitarias disponibles en el mundo del trabajo.

El estudio que se presenta tuvo como objetivo aportar a la comprensión de los pilares de construcción identitaria en el trabajo flexible chileno, a partir de las significaciones de aquellos trabajadores que, dado su nivel de calificación y su posición dentro de las organizaciones, están más expuestos a los discursos que promueven tanto la identificación con la empresa como la responsabilización en torno a la propia trayectoria. Son precisamente quienes diseñan e implementan sistemas de gestión en empresas inmersas en el mercado del trabajo flexible, quienes ejercen poder o tienen a su cargo a otras personas, los que mayormente se ven invitados a apropiarse de las exigencias y desafíos de la ideología managerial (Aubert \& De Gaulejac, 1991; Boltansky \& Chiapello, 1999; Sennett, 1998). El interés estuvo puesto en situar las narrativas acerca de las trayectorias en el marco de los escenarios de trabajo en que se desarrollaron, es 
decir, en las características de la situación de empleo, el diseño del trabajo y la gestión de personas que desarrolla la organización. A partir de estos pilares, la pregunta de investigación buscó identificar cuáles son las significaciones de los sujetos acerca de sí mismos y de sus trayectorias, su disponibilidad a la flexibilidad y sus expectativas acerca de las obligaciones mutuas en el intercambio profesional con las empresas.

\section{Método}

Se realizó un estudio de naturaleza cualitativa, de carácter analítico relacional, inspirado en los principios de la grounded theory, desarrollada en los años 60 a partir de los trabajos de Anselm Strauss y Barney Glaser (Glaser \& Strauss 1967; Strauss, 1992a, 1992b; Strauss \& Corbin, 1990). Este modelo de investigación tiene como propósito construir procesos de teorización en los que el investigador descubre o manipula categorías abstractas y genera relaciones entre ellas, a partir de ciertas estrategias fundamentales, como el método de la comparación constante, el muestreo teórico y un paradigma de codificación que permite asegurar el desarrollo y la densidad conceptual (Baszanger, 1992).

\section{Participantes}

La muestra estuvo constituida por nueve profesionales, seis hombres y tres mujeres, de entre 28 y 36 años, de la ciudad de Santiago de Chile, que trabajan en empresas flexibles o desarrollan proyectos inmersos en ambientes flexibles. Se focalizó en profesionales menores de 40 años, dado que este grupo se insertó y socializó en el mundo del trabajo luego de las reformas estructurales chilenas de empleo, en un período en el cual ya se han sentado las bases del discurso ideológico del management. Las profesiones de los entrevistados son ingeniería comercial (6), ingeniería civil (2) y derecho (1).

Los participantes fueron contactados a partir de informantes clave, en base a un perfil construido progresivamente a medida que se desarrolló el análisis, siguiendo la lógica del muestreo teórico (Strauss \& Corbin, 1990).

El análisis preliminar de las narrativas acerca de las trayectorias en contexto de empresa llevó a focalizarse también en aquellas que se desarrollan fuera del contexto de empresa. Del total de la muestra, cinco de los entrevistados trabajan en contexto de empresa y cuatro en contexto independiente.

\section{Instrumento}

La recolección de la información se hizo a través de entrevistas individuales en profundidad (Gaínza, 2006), en las que la interacción entre entrevistado/a y entrevistador/a ofrece potencialidad heurística y productiva (Blanchet \& Gotman, 2001). Para orientar la conversación se diseñó una matriz de reconstrucción de trayectorias laborales, que pedía identificar momentos relevantes de estas, en torno a los cuales se caracterizaba la situación de empleo, organización y los sistemas de gestión que regulaban el trabajo de la persona.

\section{Procedimiento}

Las entrevistas, de aproximadamente dos horas de duración, fueron realizadas por uno o dos miembros del equipo de investigación en el propio lugar de trabajo o en algún lugar público que permitiera la conversación.

De acuerdo a los términos acordados con los entrevistados y explicitados en la carta de consentimiento informado aprobada por el Comité de Ética de la Universidad Alberto Hurtado, las entrevistas fueron grabadas y luego transcritas. Para garantizar el anonimato, a cada entrevistado/a se le asignó un nombre ficticio y se eliminaron las referencias que permitieran identificar tanto a los sujetos como a las empresas en las que trabajaban. 


\section{Análisis de Datos}

El análisis de la información se realizó según el método de comparación continua (Strauss \& Corbin, 1990) e implicó procedimientos sistemáticos de codificación para sistematizar propiedades e hipótesis acerca del fenómeno estudiado, a partir del principio de la saturación teórica (Glaser \& Strauss, 1967). La primera fase, de categorización abierta, permitió obtener categorías descriptivas de información a través de un doble proceso de fragmentación y agrupación de los datos de las entrevistas. Luego, estas categorías emergentes fueron progresivamente integradas y contrastadas en el proceso de codificación axial, el cual arrojó un total de siete grandes categorías temáticas, cada una de las cuales agrupó y vinculó diferentes contenidos relacionados con la pregunta de investigación. Finalmente, el proceso de codificación selectiva integró estas categorías principales a partir de una relectura de los datos, permitiendo obtener un esquema teórico mayor. A continuación se presentan dos temáticas centrales resultantes del proceso de codificación selectiva: la experiencia de trabajo y la concepción de las propias trayectorias profesionales por parte de los entrevistados.

\section{Resultados}

Se presentan dos ámbitos de resultados estrechamente vinculados entre sí: por una parte, el referido a la experiencia de trabajo de los profesionales y, por otra, la progresiva centralidad que adquiere la movilidad al momento de concebir las propias trayectorias laborales y las estrategias desarrolladas para asegurarla. Se insertan algunas citas de las entrevistas, que permiten dar cuenta de la expresión específica de los contenidos.

\section{La Experiencia de Trabajo Centrada en el Corto Plazo}

Las narrativas de los profesionales acerca de sus trayectorias dejan traslucir una concepción de la actividad de trabajo estructurada en una temporalidad de corto plazo, centrada en responder a desafíos inmediatos.

Quienes trabajan en el contexto de empresa tienden a hablar del trabajo y de sí mismos en el trabajo a partir de los objetivos que la institución define en breves temporalidades. Los sujetos se apropian en sus narraciones de la lógica de metas individuales y colectivas que les rigen, las cuales aluden a resultados específicos de proceso, de negocio o de determinados proyectos. El cumplimiento de estas metas sirve a los sujetos no solo para narrar el propio trabajo y estructurar su experiencia cotidiana sino, además, como medida y referente del propio valor en el trabajo y de las comparaciones con los demás:

De hecho, hoy por hoy somos el mejor segmento del país. Desde el año 2007 a la fecha, este es el mejor segmento de todo el pais y me atrevería a decir este es el mejor segmento entre [Banca] Personas y [Banca] Empresa (...) somos el mejor segmento del pais, ha sido reconocido y estamos tranquilos con eso, y una de las razones por las que estamos trabajando a esta hora es justamente por eso, somos muy intensivos en trabajo (Hugo, 16-24) ${ }^{1}$

El foco en el logro de metas, ya sea en el contexto de empresa o de independencia, justifica la alta intensidad con que recurrentemente se caracteriza al propio trabajo y da sentido a la actitud de alta disponibilidad con que los profesionales lo enfrentan. Sus descripciones del propio trabajo aluden sistemáticamente a extensas jornadas —utilizando tiempos identificados por ellos mismos como propios del no trabajo- y de la conexión permanente con la actividad laboral, facilitada por las nuevas tecnologías de información.

\footnotetext{
El número final de las citas se refiere a los párrafos de la transcripción de cada entrevista. Los textos entre paréntesis corresponden a preguntas de los entrevistadores o a una referencia de empresa o área que fue excluida de la transcripción para asegurar el anonimato de las fuentes.
} 
Como consecuencia de la alta intensidad del trabajo, los entrevistados identifican procesos de acumulación de desgaste vinculado a sus trayectorias profesionales, lo que refiere a la experiencia individual de agotamiento y a consecuencias negativas a nivel social y familiar, producto de la vida laboral:

...Tengo 12 o 13 años de trayectoria laboral, en que lo que me pasó a mí fue que los primeros siete fueron muy vertiginosos y los últimos cinco he estado en esta empresa (...) tú te empiezas a cuestionar, te cuestionas tanto que al final también necesitas un periodo como para parar, para focalizarte en otras cosas que a lo mejor no las he tenido tan presente. Porque al final el día para todos tiene 24 horas y uno tiene que sacrificar y tiene que postergar cosas cuando tienes estos cambios... (Guillermo, 54)

En general se asume el desgaste como un costo inevitable asociado al trabajo, aunque los entrevistados de mayor edad señalan que en ciertos momentos de sus trayectorias reconocen la necesidad de producir un cambio respecto de la intensidad del trabajo y desarrollan ciertas estrategias de compensación o de prevención del desgaste acumulado. Al mismo tiempo, reconocen que nuevos desafíos profesionales les han obligado - y pueden volver a obligarles- a restablecer la alta disponibilidad frente al trabajo que ha caracterizado sus trayectorias.

La experiencia de trabajo centrada en el corto plazo se traduce en relaciones con los demás caracterizadas por la ambivalencia. Las lógicas de organización en las cuales los profesionales se desenvuelven requieren de permanente coordinación y negociación entre compañeros y con el entorno. Al mismo tiempo, suelen describirse ambientes de alta competencia y conflictividad interpersonal, agudizados por las continuas transformaciones organizacionales difíciles de administrar a nivel personal y grupal. Se constatan formas de vínculo social que tienden a ser instrumentales y pasajeras, en las que resalta la ambivalencia con que los sujetos adhieren y al mismo tiempo se distancian de los discursos y dispositivos de gestión que promueven trabajo colectivo y asertividad en las interacciones:

Tienen como el tema gringo de la empresa, que es el tema del servicio al cliente, de estándares de calidad, normas ISO... como eso de expresar los problemas que uno tiene, de tener la confianza como para decir a la jefatura "oye, tengo tal problema", como abierto (...) se incentiva eso, de tener una comunicación abierta, independiente de los estratos (...) se incentiva eso como la participación, como la comunicación abierta entre los empleados. (...) La gente tiene confianza como para si hay algún problema, expresarlo o informarlo (...) yo creo que funciona, funciona bien, no sé si me siento tan parte o no, yo creo que lo utilizo de vez en cuando y funciona bien (Malaquías, 406-418)

La relación con la empresa, según se desprende en las narrativas de los entrevistados, también tiende a ser ambigua. Los sujetos se apropian del lenguaje y de los objetivos organizacionales, pero en las representaciones de sí mismos se observan pocas referencias relativas a la apropiación y valoración de la imagen de la empresa. Mientras más móvil se plantean las trayectorias, la empresa se concibe como un espacio de transición e intercambio de corto plazo y no como un universo social de pertenencia.

\section{La Creciente Importancia de la Movilidad en las Trayectorias Laborales}

Las narrativas acerca de las trayectorias laborales que se han desarrollado en el contexto de empresa se caracterizan por el interés fundamental y permanente de los sujetos por la proyección, término que agrupa a una serie de otros conceptos relacionados - ascender, desarrollarse - que aluden finalmente a avanzar en puestos de importancia creciente y de mejores niveles de renta. Las posibilidades de proyección o desarrollo constituyen el criterio más recurrentemente utilizado para valorar los trabajos disponibles o deseados en relación a las propias trayectorias:

El éxito para mí es la proyección que tienes (...) para mí es mucho más importante la proyección. Por ejemplo, si tengo un amigo que está ganando cinco palos y está en una empresa chica que no tiene mucho pa' donde crecer, lo encuentro mucho menos exitoso que otros que están en tres, pero que pintan para algo mucho mejor y que en tres años más es probable... (Cristián, 178) 
El interés por la proyección toma formas diferentes en función de dos polos, uno centrado en la estabilidad y otro en la movilidad, el segundo de los cuales tendería a imponerse progresivamente. El polo de la estabilidad se refiere a la expectativa de desarrollar carrera en una empresa única, apostando a proyecciones en el largo plazo, lo que justificaría la postergación de expectativas de recompensas más inmediatas. Los sujetos confían en que la organización sabrá identificar y recompensar el buen desempeño en el largo plazo, a través de ofertas significativas de promoción, lo que les lleva a centrarse en su desempeño de corto plazo — que mezcla el deseo de demostrar méritos y cuidar el empleo-, sin elaborar estrategias específicas para la proyección al interior de la empresa:

Me dan muchas ganas de seguir en el banco, de surgir. Obviamente, como la rotación es tan alta en el banco, por la presión también, es una manera de seguir adelante, porque si la rotación es alta siempre hay puestos en los que uno puede optar y mi proyección es a corto-mediano plazo. No estar más en ese puesto por dos a tres años (Daniel, 85)

Este anhelo de proyección con estabilidad se observa directamente en las narraciones de los entrevistados más jóvenes acerca de sus expectativas actuales, así como en las narrativas de los entrevistados de mayor edad acerca de sus expectativas en etapas iniciales de sus trayectorias. Según se observa, el anhelo de estabilidad se enfrenta progresivamente a experiencias de ruptura, por lo que este polo de significación parece ir desapareciendo progresivamente a medida que los sujetos avanzan en sus trayectorias y se enfrentan a la realidad de las empresas y del mercado:

A esta altura uno las huevadas se las toma con Andina, no es tan traumático como era antes. Antes era el Banco (X), mi único trabajo, por el cual había logrado todo lo que tenía... era mi familia, era mi mundo. Y llega un huevón con plata y te compra, y empieza a echar a tus amigos, y vos quedai helado. "¿Y estos huevones quiénes son?”, y ahora yo soy tu jefe y ahora vas a trabajar en esto. (Hugo, 376)

En el polo contrario, y más generalizadamente, los entrevistados conciben sus trayectorias en el marco de la movilidad. En estos casos las expectativas de proyección y reconocimiento se hacen mucho más acotadas en el tiempo y se fundan en una concepción de la propia trayectoria dentro de un mercado amplio y no restrictivo a la empresa en la que están en el presente. El foco permanente de atención es el mercado laboral, cuyas ofertas y oportunidades tienden a jerarquizarse en base a una complejidad de criterios, entre los cuales destacan las rentas y las posibilidades de desarrollo futuro que ofrece la empresa (cuestión que finalmente las acercaría a las lógicas de trayectoria estable en empresa):

...es que pasas de división en alguna forma, pasas de jugar en segunda, pasas a jugar en primera ¿cachai? (¿Tú te sientes trabajando ahora en primera división?) Sí, pero también me gustaría trabajar o jugar en la liga española ¿̇cachai?... Hoy día juego acá en primera, pero estoy en chico ya, en Argentina. Sí, quiero ponerle, pero... Entonces acá es como que tú saltas de división, efectivamente te pegas un salto en tu desarrollo ejecutivo... (Guillermo, 194-196)

La concepción de las propias trayectorias desde el polo de la movilidad se estructura en base a representaciones bastante difusas respecto del largo plazo. Por el contrario, los sujetos muestran claridad acerca de las estrategias que implementan en el corto plazo, que permitirían abrir oportunidades para la trayectoria futura. Se han identificado tres tipos de estrategias que los sujetos combinan para posicionarse en sus lugares de trabajo y proyectarse a futuro.

La primera de ellas es la apertura al aprendizaje, que se refiere a adquirir y desarrollar la mayor cantidad de conocimientos y habilidades posibles en cada trabajo, a partir de la experiencia práctica y del modelaje de figuras relevantes (las jefaturas), los cuales no se adquirirían en instancias formales de formación. El interés por aprender se desarrollaría desde etapas muy tempranas de las trayectorias y se asocia a la expectativa general de aplicación futura de los aprendizajes: 
Sí yo no aprendo del jefe... o sea, si tu tenís un jefe que no lo admirai, o que no aprendís de él, así, yo creo que es muy frustrante (...). Igual donde estés, si estai en una organización... igual estai viendo, o sea, ponte tú, qué hace tu jefe (...). Por eso yo creo que es súper importante que admires al jefe y aprender de él, porque si no, como que lata, yo encuentro que pa' mí trabajar en una organización en la que yo no aprenda, ya sea del jefe o de alguien más, encuentro que es perder tiempo (Sofía, 148-165)

La segunda estrategia vinculada a la movilidad es frecuentemente descrita por los entrevistados como la vitrina. Se refiere a gestionar la propia imagen ante los demás, hacer visible el buen desempeño, resaltar las propias fortalezas y los aprendizajes acumulados. A esta estrategia se le atribuye legitimidad en la medida que se acompañe de un buen desempeño. De lo contrario, genera tensiones en las relaciones y críticas a quienes no acompañan su discurso de buenos comportamientos reales:

...pero tú ves para al lado y tú dices "bueno, o sea, hizo su pega bien, digamos, pero la vendió como que fuera el triple". Y yo más que darme como incomodidad un poquitito, envidio tener esa capacidad (...). Tú ves que tienen una llegada súper distinta o que son capaces de mostrar los mismos logros de una manera completamente distinta y cuando conversas con ellos... (...) se aprenden quizás distintas técnicas, pero hay un tema de personalidad que es distinto (Pamela, 425-431)

Finalmente, los profesionales que conciben sus trayectorias desde la lógica de la movilidad establecen estrategias para generar y mantener redes de contactos, que sirvan tanto para enfrentar situaciones en el trabajo presente como posibles oportunidades futuras para la movilidad:

Los contactos, yo me preocupo de cada cierto tiempo llamarlos, no perder las relaciones, de eso me preocupo harto, no es que sea un gallo interesado y me hago amigos de gallos porque el día de mañana... no, eso no me interesa, pero sí de tener una relación. (...) Y en este caso se dio de esa forma, uno busca esas situaciones, podís tener harta suerte, pero uno las busca. (...) Yo cada vez me doy cuenta que es más importante el tema de los contactos. (Cristián, 119-121)

Es posible plantear una transición general desde lógicas de proyección estable en las empresas hacia lógicas basadas en la movilidad. Este cambio se expresaría, por una parte, en la generalización de la exigencia de disposición al sacrificio, que en la lógica estable es considerada una actitud de aceptación de las exigencias laborales, requerida fundamentalmente en las primeras etapas de la trayectoria laboral, que permite proyectarse y obtener legitimidad en la trayectoria. Por el contrario, en la lógica móvil la disposición al sacrificio constituye una exigencia permanente, requerida para enfrentar cada nuevo desafío laboral.

Así, también, resulta recurrente el planteamiento de los entrevistados acerca de que los jóvenes profesionales que actualmente entran a sus empresas — a quienes deben supervisarmanifestarían una fuerte tendencia a la movilidad, expresada en expectativas muy rápidas de aumento salarial y promoción, acompañadas de la posibilidad (leída como amenaza) permanente de partir a otra empresa. Es precisamente en torno a estos profesionales más jóvenes que los entrevistados materializan una discusión central en torno a la movilidad, referida a los criterios y tiempos mínimos de permanencia en una empresa. Según varios entrevistados, los nuevos profesionales tienden a ser menos leales con la empresa y estarían comparativamente menos dispuestos al sacrificio en el inicio de sus trayectorias:

Estos cabros son más exitistas de lo que éramos nosotros, tienen expectativas mucho más altas de las que teníamos nosotros (...) si yo, para ser agente, tuve que ser el mejor ejecutivo cuatro años en regiones. Es otro mundo, acá si un huevón no es jefe de grupo, no es ni medio, es penca, si un huevón no es agente a los dos o dos años y medio, se fue a otro banco a lucrar, digamos, porque ganan más afuera ìte fijai? Entonces ellos tienen un concepto de lealtad bastante más bajo y el beneficio tiene que ser necesariamente para ellos de inmediato, porque el mercado acá lo condiciona eso. (Hugo, 334) 
Cabe señalar que esta mayor tendencia a la movilidad en los profesionales más jóvenes impone problemas concretos para la gestión de talentos en las empresas, principalmente vinculados a la retención y a las lógicas de recompensa:

(¿Y esta pega la ven cómo...?) Como algo súper pasajero, o sea un año, con suerte, estar aquí. (...) “...no, es que llevo un año y no ha cambiado nada, entonces, necesito otra cosa” me decía. Entonces, claro, hay mucha gente también que llevaba ocho meses, un año en la otra empresa, no los habian cambiado de cargo, no les habian cambiado renta y estaban también en este proceso de búsqueda (...) Cuando yo trato de hablar, digamos, para arriba y decir, "oye, es que o les ofrezco más plata, o se me van”. O sea, si queremos retener a esta persona, necesito más plata y si no... (...) Me dicen (desde la empresa) "pero cómo - me dicen-y los valores de entrar a una empresa para adquirir experiencia, para aprender, donde el objetivo último es aprender, y no ascender, ni conseguir un sueldo, ni nada, ¿dónde están esos valores?, ¿dónde están como esos objetivos de vida?". Y yo digo "no existen." (Pamela, 373-375)

\section{Discusión}

Los resultados expuestos permiten caracterizar con especial claridad las narraciones de los profesionales que representan una elite dentro de contextos organizacionales caracterizados por la sofisticación de las pautas de organización, de movilización subjetiva y de gestión del empleo propias del trabajo flexible. La evidencia fundamental se refiere a la consolidación de lógicas de construcción identitaria en el trabajo basadas en la movilidad permanente. Esto se constata tanto en las narrativas acerca de las propias trayectorias, en la que los sujetos muestran una progresiva aceptación de la movilidad, como en las percepciones de los entrevistados acerca de los nuevos referentes con que se manejan los jóvenes profesionales que hoy entran al mundo laboral, los cuales habrían adoptado las prácticas discursivas de la movilidad desde antes de entrar al mundo del trabajo.

Concretamente, es posible plantear una profunda transformación de los ejes de construcción identitaria que desarrollan los profesionales en el trabajo, caracterizados por la individualización de su trayectoria, la aceptación de la movilidad permanente y de las estrategias y disposiciones que esta exige, así como por la focalización casi exclusiva en el corto plazo, para lo cual se aceptan los referentes de medición y comparación establecidos por la empresa.

Esta transformación, sin embargo, no es simple ni está exenta de tensiones. El juicio crítico de los entrevistados hacia la menor lealtad y disposición al sacrificio de los profesionales recién egresados da luces sobre una cierta nostalgia de la estabilidad y de la proyección en el largo plazo dentro de la empresa. Los profesionales más antiguos proyectarían en los más jóvenes el cambio de paradigma en torno a las trayectorias laborales y su crítica hacia ellos condensaría el malestar que acumulan en torno a la movilidad y sus exigencias de disponibilidad.

Queda en evidencia, además, el desajuste actual de los sistemas de recompensa y retención de las empresas y se torna interesante atender a la forma en que estas reaccionarán en el mediano plazo a fin de desarrollar sistemas más efectivos de retención de nuevos cuadros y gestión del talento.

En relación a las diferentes orientaciones que proponen los discursos y prácticas de gestión identificados en la revisión conceptual, es posible plantear que los profesionales adhieren fundamentalmente a las prácticas discursivas que enfatizan la individualidad y la responsabilización por sobre disposiciones más cercanas a la identificación cultural con la empresa. La construcción identitaria a partir de la movilidad se realiza en el marco de los dispositivos de gestión pero, de todos modos, se acompañan actitudes de distancia o cinismo en la implicación con la empresa y en la relación con los demás.

De todas formas, la proyección individual como principal foco de atención de los profesionales parece lejos del ideal de auto-realización anunciado pomposamente en los discursos de las empresas. Este estudio muestra que la búsqueda de proyección y desarrollo no se orienta a la realización personal, sino que fundamentalmente a obtener roles mejor pagados y de mayor 
estatus dentro de un mercado de trabajo representado como estructurado y jerarquizado. El desafío permanente de empleabilidad exige descifrar las pistas de esa estructura, correr riesgos y acumular desgaste en movilizar masiva e intensamente los recursos disponibles —uno mismo - en la tarea. No existen proyecciones de largo plazo en torno al contenido o al impacto del trabajo que den un sentido más trascendente a la experiencia ni se observan críticas a las condiciones sociales a la base de su sufrimiento e insatisfacción laboral presente.

Los planteamientos aquí formulados deben entenderse como una aproximación a las transformaciones identitarias en el trabajo contemporáneo, específicamente referidas a los profesionales de empresas flexibles. La estrategia metodológica implementada utilizó exclusivamente narraciones de los entrevistados acerca de sus trayectorias, lo que plantea la limitación de enfrentarse a discursos excesivamente estructurados, que esconden los espacios de tensión e incoherencia a partir de los cuales se construyen precisamente las identidades en el trabajo. Pese a que se buscó circunscribir las narrativas a los escenarios del trabajo en los cuales se desarrollaron las trayectorias, existen limitaciones ciertas para el acceso a la dimensión relacional de las identidades laborales, lo que plantea nuevos desafíos para la investigación en torno al tema.

Los hallazgos deben circunscribirse a un grupo particular dentro de las empresas chilenas: los profesionales de alta calificación que tienen acceso a posibilidades de reconocimiento — de construcción identitaria - en la empresa. Como se ha abordado en otros trabajos empíricos (Espinoza \& Soto, 2008), trabajadores más periféricos a la generación de valor también estructuran narrativas en torno a la búsqueda del "desarrollo" y la movilidad, aunque en esos casos estos referentes aluden más bien a la expectativa de escapar de situaciones de precariedad hacia condiciones básicas de estabilidad y seguridad del empleo.

\section{Referencias}

Abramo, L., Montero, C. \& Reinecke, G. (1997). Cambio tecnológico, encadenamientos productivos y calificaciones del trabajo en Chile: un balance. En M. Novick \& M. Gallart (Comp.), Competitividad, redes productivas y competencias laborales: homogeneidad o segmentación? (pp. 145-191). Montevideo, Uruguay: Organización Internacional del Trabajo, Centro Interamericano para el Desarrollo del Conocimiento en la Formación Profesional.

Acuña, E. (2005). Trayectorias laborales: el tránsito entre el trabajo asalariado y el empleo independiente (Cuadernos de Investigación 23). Santiago, Chile: Dirección del Trabajo.

Acuña, E. (2008). Flexibilidad laboral: experiencias de trabajadores chilenos. En A. Soto (Ed.), Flexibilidad laboral y subjetividades: hacia una comprensión psicosocial del empleo contemporáneo (pp. 61-77). Santiago: LOM/ Universidad Alberto Hurtado.

Alcover, C. (2002). El contrato psicológico, el componente implícito de las relaciones laborales. Málaga, España: Aljibe.

Alvesson, M., Ashcraft, K. L. \& Thomas, R. (2008). Identity matters: Reflections on the construction of identity scholarship in organization studies. Organization, 15, 5-28. doi:10.1177/1350508407084426. Extraído de http:// org.sagepub.com

Alvesson, M. \& Robertson, M. (2006). The best and the brightest: The construction, significance and effects of elite identities in consulting firms. Organization, 13, 195-224. doi:10.1177/1350508406061674. Extraído de http://org. sagepub.com

Alvesson, M. \& Willmott, H. (2002). Identity regulation as organizational control: Producing the appropriate individual. Journal of Management Studies, 39, 619-644. doi:10.1111/1467-6486.00305

Aubert, N. \& De Gaulejac, V. (1991). Le coût de l'excellence [El costo de la excelencia]. Paris, Francia: Éditions du Seuil.

Baszanger, I. (1992). Les chantiers d'un interactionniste américain [Las canteras de un interaccionismo americano]. En I. Baszanger (Ed.), La trame de la négociation: sociologie qualitative et interactionnisme [La trama de la negociación: sociología cualitativa e interaccionismo] (pp. 11-63). Paris, Francia: L’Harmattan.

Blanchet, A. \& Gotman, A. (2001). L'enquête et ses méthodes: l'entretien [La investigación y sus métodos: la entrevista]. Paris, Francia: Nathan.

Boltansky, L. \& Chiapello, E. (1999). Le nouvel esprit du capitalisme [El nuevo espíritu del capitalismo]. Paris, Francia: Gallimard.

Contreras, D. (1999). Distribución del ingreso en Chile: nueve hechos y algunos mitos. Perspectivas, 2, 211-332.

Courpasson, D. (1997). Régulation et gouvernement des organisations, pour une sociologie de l'action managériale [Regulación y gobierno de las organizaciones, para una sociología de la acción gerencial]. Sociologie du Travail, 1, 39-61. 
Courpasson, D. (2000). L'action contrainte, organisations libérales et domination [La acción obligada, organizaciones liberales y dominación]. Paris, Francia: Presses Universitaires de France.

Down, S. \& Reveley, J. (2009). Between narration and interaction: Situating first-line supervisor identity work. Human Relations, 62, 379-401. doi:10.1177/0018726708101043. Extraído de http://hum.sagepub.com

Dubar, C. (1991). La socialisation, construction des identités sociales et professionnelles [La socialización, construcción de identidades sociales y profesionales]. Paris, Francia: Armand Colin.

Dubar, C. (1992). Formes identitaires et socialisation professionnelle [Formas identitarias y socialización profesional]. Revue Française de Sociologie, 33, 505-529.

Dubar, C. (2000). La crise des identités, l'interprétation d'une mutation [La crisis de las identidades, la interpretación de una mutación]. Paris, Francia: Presses Universitaires de France.

Echeverría, M. (2009). La historia inconclusa de la subcontratación y el relato de los trabajadores. Santiago, Chile: Dirección del Trabajo.

Elchardus, M. \& Smits, W. (2008). The vanishing flexible: Ambition, self-realization and flexibility in the career perspectives of young Belgian adults. Work Employment and Society, 22, 243-262. doi:10.1177/0950017008089103. Extraído de http://wes.sagepub.com

Enriquez, E. (1997a). Les jeux du pouvoir et du désir dans l'entreprise [Los juegos del poder y del deseo en la empresa]. Paris, Francia: Desclée de Brouwer.

Enriquez, E. (1997b). L’organisation en analyse [La organización en análisis]. Paris, Francia: Presses Universitaires de France.

Enríquez, A. \& Rentería, E. (2007). Estrategias de aprendizaje para la empleabilidad en el mercado del trabajo de profesionales recién egresados. Universitas Psychologica, 6, 89-103.

Espinoza, G. \& Soto, A. (2008). El vínculo que establece el trabajador(a) con la empresa flexible: el caso de una empresa de servicios. En A. Soto (Ed.), Flexibilidad laboral y subjetividades: hacia una comprensión psicosocial del empleo contemporáneo (pp. 279-309). Santiago, Chile: LOM/ Universidad Alberto Hurtado.

Everaere, C. (1999). Emploi, travail et efficacité de l'entreprise: les effets pervers de la flexibilité quantitative [Empleo, trabajo y eficacia en la empresa: los efectos perversos de la flexibilidad cuantitativa]. Revue Française de Gestion, Juillet-Août, 5-21.

Gadrey, J. (1996). Service: la productivité en question [Servicio: la productividad en cuestión]. Paris, Francia: Desclée de Brouwer.

Gaínza, A. (2006). La entrevista en profundidad individual. En M. Canales (Ed.), Metodologías de investigación social (pp. 219-263). Santiago, Chile: LOM.

García, C. \& Carvajal, L. (2007). Tecnologías empresariales del yo: la construcción de sujetos laborales en el contexto del trabajo inmaterial. Universitas Psychologica, 6, 49-58.

Gazier, B. (2003). Tous "sublimes", vers un nouveau plein emploi [Todos "sublimes", hacia un nuevo empleo pleno]. Paris, Francia: Flammarion.

Ghiardo, F. \& Dávila, O. (2008). Trayectorias sociales juveniles: ambivalencias y discursos sobre el trabajo. Santiago, Chile: Instituto Nacional de la Juventud/CIDPA Centro de Estudios Sociales.

Glaser, B. G. \& Strauss, A. L. (1967). The discovery of grounded theory: Strategies for qualitative research. New York, NY: Aldine.

Grote, G. \& Raeder, S. (2009). Careers and identity in flexible working: Do flexible identities fare better? Human Relations, 62, 219-244. doi:10.1177/0018726708100358. Extraído de http://hum.sagepub.com

Goffman, E. (2001). La presentación de la persona en la vida cotidiana. Buenos Aires, Argentina: Amorrortu.

Goffman, E. (2003). Estigma: la identidad deteriorada. Buenos Aires, Argentina: Amorrortu.

Hall, S. (2003). ¿Quién necesita 'identidad’? En S. Hall \& P. Dugay (Comp.), Cuestiones de identidad cultural (pp. 1339). Buenos Aires, Argentina: Amorrortu.

Hall, D. T. \& Chandler, D. E. (2005). Psychological success: When the career is a calling. Journal of Organizational Behavior, 26, 155-176. doi:10.1002/job.301

Infante, R. \& Sunkel, O. (2009). Chile: hacia un desarrollo inclusivo. Revista CEPAL, 97, 135-154. Extraído de http:// www.eclac.org/publicaciones/xml/3/35853/RVE97InfanteSunkel.pdf

Kosmala, K. \& Herrbach, O. (2006). The ambivalence of professional identity: On cynicism and jouissance in audit firms. Human Relations, 59, 1393-1428. doi:10.1177/0018726706071526. Extraído de http://hum.sagepub.com

Le Goff, J. P. (1995). Le mythe de l'entreprise: critique de l'idéologie managériale [El mito de la empresa: crítica a la ideología managerial]. Paris, Francia: La Découverte.

López, D. (2008). Flexibilidad laboral en Chile: un paseo por la realidad. En A. Soto (Ed.), Flexibilidad laboral y subjetividades: hacia una comprensión psicosocial del empleo contemporáneo (pp. 41-50). Santiago, Chile: LOM/ Universidad Alberto Hurtado.

Martínez, G., De Cuyper, N. \& De Witte, H. (2010). Review of temporary employment literature: Perspectives for research and development in Latin America. Psykhe, 19(1), 61-73. doi:10.4067/S0718-22282010000100005

Maruani, M. \& Reynaud, E. (1993). Sociologie de l'emploi [Sociología del empleo]. Paris, Francia: La Découverte.

Mauro, A. (2004). Trayectorias laborales en el sector financiero. Recorridos de las mujeres. Santiago, Chile: Naciones Unidas, CEPAL.

Mauro A. \& Yáñez, S. (2005). Trayectorias laborales y previsión social en Chile en un contexto de flexibilidad (Cuadernos de Investigación 1). Santiago, Chile: Centro de Estudios de la Mujer.

Morrison, E. W. \& Robinson, S. L. (1997). When employees feel betrayed: A model of how psychological contract violation develops. Academy of Management Review, 22, 226-256.

Raineri, A. (2001). Administración del cambio organizacional en empresas chilenas. Estudios de Administración, 8(2), 1-41. 
Rentería, E. \& Malvezzi, S. (2008). Empleabilidad, cambios y exigencias psicosociales en el trabajo. Universitas Psychologica, 7, 319-334.

Rousseau, D. M. (1995). Psychological contracts in organizations: Understanding written and unwritten agreements. Thousand Oaks, CA: Sage.

Sainsaulieu, R. (1977). L’identité au travail [La identidad en el trabajo]. Paris, Francia: Presses de la Fondation Nationale des Sciences Politiques.

Sennett, R. (1998). La corrosión del carácter: las consecuencias personales del trabajo en el nuevo capitalismo. Barcelona, España: Anagrama.

Sisto, V. \& Fardella, C. (2009). Control narrativo y gubernamentalidad: la producción de coherencia en las narrativas identitarias. El caso de profesionales chilenos adultos jóvenes en condiciones de vinculación laboral flexible. Forum Qualitative Sozialforschung / Forum: Qualitative Social Research, 10(2), Artículo 29. Extraído de http:// nbn-resolving.de/urn:nbn:de:0114-fqs0902292

Soto, A. (2009). Formas y tensiones de los procesos de individualización en el mundo del trabajo. Psicoperspectivas, 8, 102-119. Extraído de www.psicoperspectivas.cl

Soto, A., Espinoza, G. \& Gómez, J. (2008). Los aspectos subjetivos de la flexibilidad laboral. En A. Soto (Ed.), Flexibilidad laboral y subjetividades: hacia una comprensión psicosocial del empleo contemporáneo (pp. 11-37). Santiago, Chile: LOM/Universidad Alberto Hurtado.

Strauss, A. (1992a). Une perspective en termes de monde social [Una perspectiva en términos del mundo social]. En I. Baszanger (Ed.), La trame de la négociation: sociologie qualitative et interactionnisme [La trama de la negociación: sociología cualitativa e interaccionismo] (pp. 269-282). Paris, Francia: Éditions L'Harmattan.

Strauss, A. (1992b). Négociations: introduction à la question [Negociaciones: introducción a la cuestión]. En I. Baszanger (Ed.), La trame de la négociation: sociologie qualitative et interactionnisme [La trama de la negociación: sociología cualitativa e interaccionismo] (pp. 11-63). Paris, Francia: Éditions L'Harmattan.

Strauss, A. L. \& Corbin, J. (1990). Basics of qualitative research: Grounded theory procedures and techniques. Newbury Park, CA: Sage.

Sveningsson, S. \& Alvesson, M. (2003). Managing managerial identities: Organizational fragmentation, discourse and identity struggle. Human Relations, 56, 1163-1193. doi:10.1177/00187267035610001. Extraído de http://hum. sagepub.com

Fecha de recepción: Octubre de 2010.

Fecha de aceptación: Marzo de 2011. 